\title{
Improving Energy Saving in Wireless Systems by Using Dynamic Power Management
}

\author{
Carla-Fabiana Chiasserini, Member, IEEE, and Ramesh R. Rao, Senior Member, IEEE
}

\begin{abstract}
In this paper, we develop a novel approach to conserving energy in battery-powered communication devices. There are two salient aspects to this approach. First, the battery-powered devices move through multiple, progressively deeper, sleep states in a predictable manner. Nodes in deeper sleep states consume lower energy while asleep, but incur a longer delay and higher energy cost to awaken. Second, the nodes are woken up on demand through a paging signal. To awaken nodes that are in deep sleep, the paging signal has to be decoded using very low power circuits such as those used in radio frequency tags. To accommodate this need, in a manner that scales well with the number of nodes, the number of distinct paging signals has to be much less than the number of possible nodes. This is accomplished through a group-based wakeup scheme, which initially awakens the targeted node along with a number of other similarly disposed nodes that subsequently return to their original sleep state. Tradeoffs among energy consumption, delay as well as overhead, are presented; comparisons with other protocols show the potential for $16 \%$ to $50 \%$ improvement in energy consumption.
\end{abstract}

Index Terms-Dynamic power management, energy efficiency, wireless quality of service $(\mathrm{QoS})$.

\section{INTRODUCTION}

$\mathbf{M}$ INIMIZING energy consumption in battery-powered devices is crucial to the design of wireless communication networks. Several protocol solutions have been proposed in the literature to reduce the energy expenditure of portable communication nodes in transmit and receive mode. In [1], the authors present energy-aware access protocols minimizing the time period during which radio frequency (RF) identification devices need to be active. In [2], a medium access control (MAC) scheme based on traffic scheduling and radio resource reservation is proposed, which allows a network node to enter low-power operational states when idle. A study of energy consumption of MAC protocols from the tranceiver usage perspective is presented in [3].

A power conservation technique that has been generally recognized as effective is discontinuous reception, whereby inactive nodes power down and turn ON their receiver at some future time instant. Paging schemes based on discontinuous reception can be classified as synchronous or asynchronous.

Manuscript received June 28, 2001; revised May 13, 2002 and August 23, 2002; accepted August 27, 2002. The editor coordinating the review of this paper and approving it for publication is Y. Fang. This work was supported by Intersil, Cal-(IT) ${ }^{2}$, and by the Centro di Eccellenza per le Radio Comunicazioni Multimediali (CERCOM), Torino, Italy.

C.-F. Chiasserini is with the Dipartimento di Elettronica, Politecnico di Torino, 10129 Torino, Italy (e-mail: chiasserini@ polito.it).

R. R. Rao is with the Electrical and Computer Engineering Department, University of California, San Diego, CA 92093 USA (e-mail: rao@cwc.ucsd.edu).

Digital Object Identifier 10.1109/TWC.2003.817445
In synchronous paging protocols, the broadcast transmission from the base station (BS) and the nodes' waking-up time instants are synchronized. Examples of such protocols are the following. The POCSAG scheme [4] uses a coding format based on batches, with each batch consisting of eight frames. A node can be paged at only one of the eight frames so that it may power down during the other seven to save energy. A similar approach is used in the FLEX [5] system where data intended for a particular pager is scheduled in a predefined time slot. In the MOBITEX data system [6] and in IEEE 802.11 [7], nodes in power saving mode wake up in time for a broadcast transmission from the BS that notifies which nodes have pending data. In high-performance radio local area network (HIPERLAN) [8], the wireless LAN standard specified by European Telecommunications Standards Institute, radio nodes that need to save power, so-called $p$-savers, communicate their own sleep-awake schedule to the so-called p-supporter node. The p-supporter queues all the packets destined to the p-savers and transmits these packets during the p-saver's active time.

On the contrary, asynchronous paging schemes do not require system synchronization and each node is free to power ON and OFF its receiver based on the battery status. An asynchronous, in-band (IB) protocol is presented in [9]. There, as the node powers ON, it keeps listening to the radio channel for a short time period. If a paging message is received, the node sends an acknowledgment to the BS; otherwise, it switches OFF again. Paging and acknowledgment messages are transmitted in-band, i.e., within the node data flow. As shown in [9], such a method outperforms the synchronous approach for light traffic loads.

Both synchronous and asynchronous paging schemes cause network nodes to become unavailable for reception from time to time, making any communication with them impossible. While this allows for energy saving, it raises several problems related to quality of service (QoS) provisioning. Therefore, a crucial issue in designing energy-efficient paging protocols is to optimally trade off between energy savings and QoS requirements on packet delay. To achieve this balance, we introduce a novel solution which employs discontinuous reception in combination with a power management policy at the network node. In particular, we make an asynchronous paging scheme and a power management algorithm interact and jointly operate to meet the system constraints.

We explain our approach in some detail in Section II and we introduce notations and outline some work related to power management in Section III. The proposed paging scheme is described in Section IV; the protocol analysis and performance are presented in Sections V and VI, respectively. Finally, Section VII concludes the paper. 


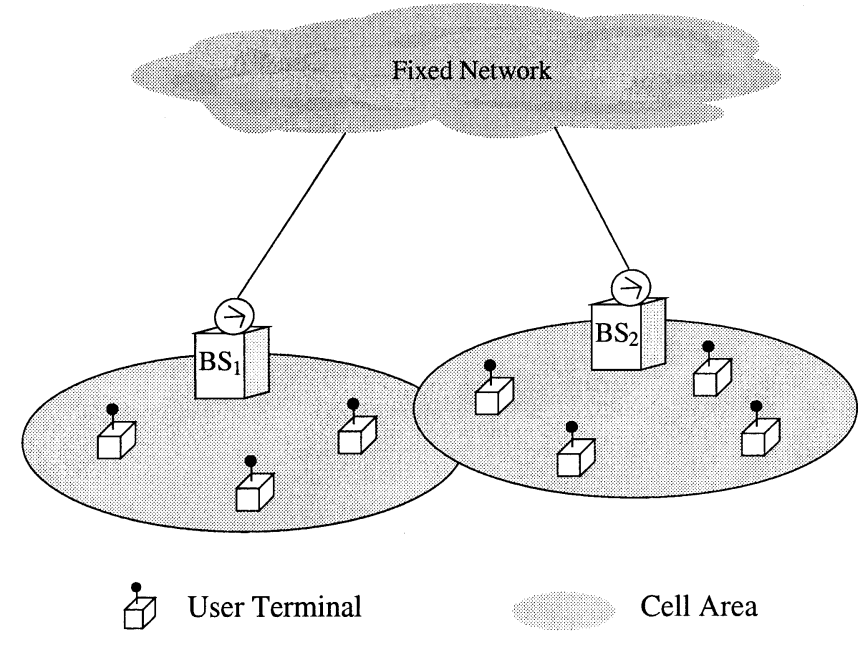

Fig. 1. Network scenario.

\section{Combining Paging With Power Management}

We focus on the packet data transfer between a BS, that is connected to the wired network, and mobile nodes. Other network scenarios, where a node is elected to be the master with respect to the other nodes in the vicinity, can be considered as well. Fig. 1 shows the reference network scenario where communication nodes, either mobile or fixed, access the fixed network and communicate among each other through the BS. The BS is responsible for collecting the uplink traffic generated by the nodes in the cell and for delivering the downlink data flow to the communication nodes. We focus on downlink traffic because it is envisioned that in future wireless systems the traffic pattern will be highly asymmetrical, with $50 / 1$ or greater ratio favoring the downlink [10].

During the idle periods, communication nodes can switch OFF parts of their circuitry (e.g., display, RF component, digital component, etc.) and enter a sleep state. Various sleep states are identified based on the associated power consumption and time to wake up; the deeper the sleep state, the less the power consumed and larger the delay overhead. In electronic systems, such as portable computers and personal digital assistants, the evolution of a device through different operational states is usually controlled by a dynamic power management (DPM) policy. A DPM technique automatically detects the system components that are idle and determine whether they have to be switched OFF [11]-[16]. The aim is to reduce power consumption without significantly degrading the device performance.

In this paper, we develop a way to tradeoff energy saving and traffic QoS degradation by exploiting the synergy between the power management policy, executed at the communication node, and the scheduling of downlink traffic at the BS. We propose a scheme where the BS tracks the power state of each node and exploits this information to adjust the transmission of downlink traffic to the node's energy constraints. If necessary, paging is delayed in order to let nodes stay in sleep state longer, and hence, increase their energy saving, provided that the required QoS is still guaranteed. Nodes do not need to power ON to see whether they have pending traffic at the BS. Instead, the BS wakes nodes when necessary. To implement this, we need

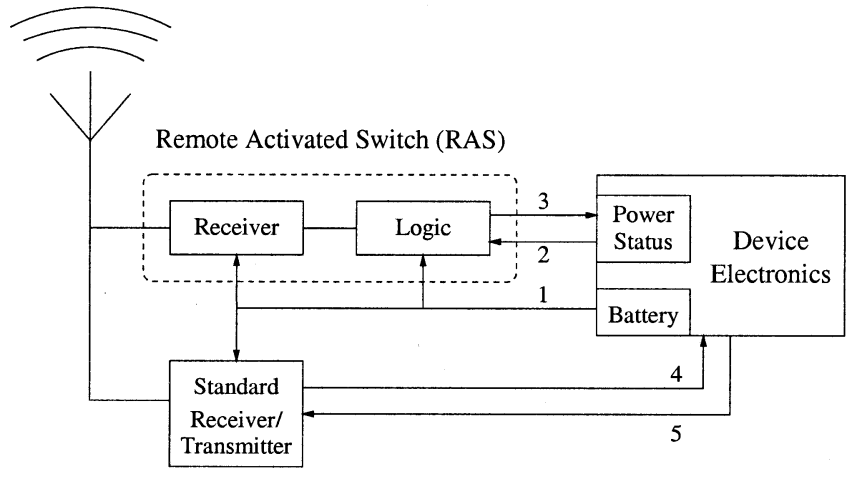

Fig. 2. Scheme of the node's device circuit.

a method for the remote activation of a communication node through an RF signal, ideally at negligible energy cost.

RF tags technology offers good examples of low power or totally passive devices that use RF power received from the BS to drive the logic and transmission parts of the circuit [17]-[20]. RF tags have been used as transmitter-receiver devices (transponders) for remote localization and identification of animals, cars, and other items [20]-[22]. The schematic representation of a switch that can be used to remotely activate the device is shown in Fig. 2. Whenever the communication node becomes idle, it enters a sleep state, i.e., the standard receiver-transmitter and parts of the device electronics are turned OFF. Paging signals are received and demodulated by the remotely activated switch (RAS). Then, the signal information is passed to the logic circuit that detects the bits sequence. If the received sequence matches the node's paging sequence, the device turns oN the standard receiver. Notice that the RAS receiver may be either totally passive (e.g., an amplitude demodulator) or supplied by the battery source through Connection 1.

\section{PRELIMINARIES}

We assume that a communication node can be in $L$ different states depending on the operational status of the system components. Each state is characterized by a certain power consumption, denoted by $P_{l}(l=1, \ldots, L)$, such that $P_{1}<P_{2}<\cdots<$ $P_{L}$. Every transition from state $l$ to state $f(l, f=1, \ldots, L$ and $f \neq l$ ) has a cost in terms of power consumption, denoted by $P_{l, f}$, and of delay overhead, denoted by $W_{l, f}$. The cost associated with transitions from state $l$ to $f$, with $l=2, \ldots, L$, and $f<l$, is usually much lower than the cost associated with the reverse transition, and for the sake of simplicity is neglected.

Now, consider a device for which the only possible operational states are ON and OFF, and moving from state ON to state OFF requires a significant amount of energy. It is clear that the device should be shut down only when its idle period can be long enough so that the saved energy compensates for the transition energy expenditure. Let us define $Z_{\text {off }}$ as the minimum time that the device has to spend in state OFF to compensate for the wakeup overhead. We must have

$$
P_{\text {off }} Z_{\text {off }}+P_{\text {off }, \text { on }} W_{\text {off }, \text { on }}=P_{\text {on }}\left(Z_{\text {off }}+W_{\text {off }, \text { on }}\right) .
$$


In (1), the left term is the sum of the energy spent by the device while being in state OFF and the cost due to the transition from state OFF to state ON. The right term is the energy expenditure corresponding to the device being in state ON all the time. From (1), it follows that

$$
Z_{\text {off }}=\max \left(0, \frac{\left(P_{\text {on }}-P_{\text {off }, \text { on }}\right) W_{\text {off }, \text { on }}}{P_{\text {off }}-P_{\text {on }}}\right) .
$$

The time period $Z_{\text {off }}+W_{\text {off,on }}$ is defined as the minimum break-even time, i.e., the minimum length of the device's idle period to save energy [12].

The discussion above can be easily generalized to the case $L>2$. Consider a node moving from state $l$ to state $f(l, f=$ $1, \ldots, L$ and $l \neq f$ ); we have

$$
Z_{l, f}=\max \left(0, \frac{\left(P_{f}-P_{l, f}\right) W_{l, f}}{P_{l}-P_{f}}\right)
$$

where $Z_{l, f}$ is the minimum time that the device has to spend in state $l$ to save energy, and $Z_{l, f}+W_{l, f}$ is the associated minimum break-even time. Note that, since we assume $W_{l, f}$ to be equal to zero for $f<l$, we have: $Z_{l, f}=0$ for $f<l$.

Clearly, a device should enter a sleep state only if this allows for energy saving, i.e., if the device's idle period can be greater than the minimum break-even time associated with the sleep state. To make this happen, a DPM technique is often applied to control the device's operational state [11]-[16]. A DPM policy must take into account the cost of a transition from an operational state to another, and should be able to foresee for how long a device will remain idle. Depending on the approach that is used, DPM policies are classified as predictive or stochastic policies.

A widely used predictive technique consists in turning OFF the system components if an idle time greater than or equal to a timeout value $T_{1}$ is detected. This approach is based on the assumption that if the idle time is greater than $T_{1}$, the system is likely to remain idle for a time period long enough to save energy. A more accurate method is proposed in [11], where the upcoming idle time is predicted by using an exponential-average approach. If the predicted idle time is sufficiently long, the system component is switched OFF at once. However, predictive techniques have a few limitations: they assume only two states for the system (ON and OFF), they cannot provide an accurate tradeoff between energy saving and performance degradation, and they do not deal with a generic system architecture where service requests can be queued.

A stochastic policy has been proposed in [23] to overcome these limitations. The considered system can enter $L$ states with $L \geq 2$, and it is composed of a service provider, a service requester, a power manager, and a request queue. The service provider and requester are represented as Markov processes, and the power manager determines the device state of operation by issuing commands to the service provider. In this case, the optimal policy strictly depends on how the system is modeled and on the abstractions that have been made. Moreover, the amount of energy consumed by the power manager remains to be accounted for.

\section{PRoposed SCHEME}

The objective of all DPM policies is to allow communication nodes to save energy by entering a sleep state while still meeting the QoS requirements on packet delay. Unlike the previous DPM policies, the proposed scheme achieves this goal by adjusting the node's idle time through an appropriate scheduling of the downlink traffic. Idle times are extended by delaying the waking-up events as long as traffic delay constraints are still satisfied. The proposed scheme combines a predictive power management technique executed at the node with a paging protocol.

\section{A. Predictive Power Management Policy}

Let us first assume $L=2$, with ON and OFF being the device's two possible states. The adopted DPM policy operates as follows.

Communication nodes are grouped into $Q$ different service classes depending on their battery status and QoS requirements on packet delay. Nodes belonging to different service classes are assigned different sleep patterns. Consider the generic node $j$ belonging to service class $q$. The sleep pattern is represented by the following values.

1) The timeout (i.e., the idle time) after which node $j$ currently in state ON enters state OFF, and the timeout after which $j$ can move from state OFF back to state ON. We denote these values with $T_{\text {on,off }}^{(q)}$ and $T_{\text {off,on }}^{(q)}$, respectively.

2) The minimum time period that $j$ has to spend in state OFF before moving to state ON. We denote this value with $Y_{\text {off }}^{(q)}$ and impose that $Y_{\text {off }}^{(q)}$ is equal to or greater than $Z_{\text {off. }}$. It follows that

$$
T_{\text {off,on }}^{(q)} \geq T_{\text {on,off }}^{(q)}+Y_{\text {off }}^{(q)} \geq T_{\text {on,off }}^{(q)}+Z_{\text {off. }}
$$

The values $T_{\mathrm{on}, \text { off }}^{(q)}, T_{\mathrm{off}, \text { on }}^{(q)}$, and $Y_{\mathrm{off}}^{(q)}$ are chosen in such a way that (4) holds, and the nodes' requirements on packet delay and energy savings are satisfied. For example, if a node has little battery capacity left, it will set $T_{\text {on,off }}^{(q)}$ to be small so that it quickly enters state OFF, and select a large $Y_{\text {off }}^{(q)}$ to increase energy savings. In this case, the node saves more energy, although the delay penalty to get back to state ON may be significant. On the contrary, if a node is concerned mainly about QoS requirements on packet delay, it will use a large $T_{\text {on,off }}^{(q)}$ to rarely enter state OFF and a small $Y_{\text {off }}^{(q)}$ to be more reactive. Moreover, the values $T_{\text {on,off }}^{(q)}$ should be selected by taking into account the traffic arrival process. For instance, in the case of bursty traffic, we may want a node to quickly enter deep sleep states as it becomes idle, since it is likely to remain so for a long time. ${ }^{1}$

For each network node, the sleep pattern can be chosen either by the node itself or by the BS, according to the QoS requirements on packet delay of the downlink connection. An appropriate message exchange can be defined to let the BS and the network node agree on the sleep pattern to use. However, both the node and the BS should be able to renegotiate the sleep pattern if necessary.

\footnotetext{
${ }^{1}$ While deriving the performance results, we will assume that the downlink packet traffic arrives at the BS according to a Poisson distribution, as in [9].
} 
The above discussion can be generalized for $L>2$ as follows.

- We denote the sleep pattern associated to service class $q$ by $\Sigma^{(q)}$. The timeout values can be defined by means of a matrix, whose entries $\sigma_{l, f}=\left\{T_{l, f}^{(q)}\right\}$ represent the time period after which a node belonging to service class $q$ and currently in state $l$ can enter state $f$, for $l, f=1, \ldots, L$ and $q=1, \ldots, Q$. For every state $l$, the next power state with power consumption lower than $P_{l}$, entered by the communication device, is deterministically assessed. The values $T_{l, f}^{(q)}$ related to impossible transitions are set to infinity. We indicate with $\tau_{j}$ the period elapsed since the last time instant the generic node $j$ was paged. We have that transitions from state $l$ to state $f$, with $f<l$, occur when $\tau_{j}=T_{l, f}^{(q)} ;$ transitions from state $l$ to state $f$, with $f>l$, can take place anytime, provided that $\tau_{j} \geq T_{l, f}^{(q)}$. We highlight that this approach leads to an extremely simple power management technique at the communication node. In fact, given the sleep pattern matrix, the node follows a deterministic pattern through the sleep states.

- Upon receiving a paging message, a device moves from its current state to a state associated with a higher power consumption. If there is only one less deep power state to which the device can move, this is uniquely determined by the sleep pattern matrix. Otherwise, it has to be specified by the BS in the paging message. For the sake of simplicity, in the following we assume that when a node is paged, it always enters state $L$, i.e., the operational state where the node is fully working.

- Equation (4) can be easily generalized by considering a node in service class $q$ moving through power states $\{b, l, f\}$. For any state $b$ with $P_{b}>P_{l}$, the time period that the node has to spend in state $l$ before moving to state $f$ must be at least equal to $Y_{l, f}^{(q)}$, with $Y_{l, f}^{(q)}$ subject to $Y_{l, f}^{(q)} \geq Z_{l, f}$. We have

$$
T_{l, f}^{(q)} \geq T_{b, l}^{(q)}+Y_{l, f}^{(q)}
$$

Observe that if $P_{l}>P_{f}, T_{l, f}^{(q)}$ is the timeout value after which the deeper sleep state, $f$ is entered from state $l$. Instead, if $P_{l}<P_{f}$, condition (5) ensures that a node exits its current power state $l$ only if its idle period has been sufficiently long to save energy.

1) An Example: Consider a sensor node consisting of a StrongARM microprocessor, a memory, an RF circuitry, and a microelectromechanical system (MEMS) with analog-digital converter. As described in [16], by taking into account that each component can have different power modes, the useful operational states are those reported in Table I [16]. States with increasing $l(l=1, \ldots, 5)$ correspond to a higher power consumption and a smaller delay overhead.

We consider the following sleep pattern matrix:

$$
\left(\begin{array}{ccccc}
\infty & \infty & \infty & \infty & T_{1,5}^{(q)} \\
T_{2,1}^{(q)} & \infty & \infty & \infty & T_{2,5}^{(q)} \\
\infty & T_{3,2}^{(q)} & \infty & \infty & T_{3,5}^{(q)} \\
\infty & \infty & T_{4,3}^{(q)} & \infty & T_{4,5}^{(q)} \\
\infty & \infty & \infty & T_{5,4}^{(q)} & \infty
\end{array}\right)
$$

TABLE I

Useful Operational States For a Sensor Node. The Microprocessor Power Modes Are Active, Idle, and Sleep. The Memory Can Be IN ACtive or SLEEP Mode. The MEMS and ANAlog-To-Digital CONVERTER CAN BE ON OR OFF. THE RF CiRCUITRY POWER MODES ARE TRANSMit (tx), RECEIVE (rx), AND OFF

\begin{tabular}{c|c|c|c|c}
\hline State & StrongARM & Memory & MEMS and A/D converter & RF \\
\hline 1 & sleep & sleep & off & off \\
\hline 2 & sleep & sleep & on & off \\
\hline 3 & sleep & sleep & on & $r x$ \\
\hline 4 & idle & sleep & on & $r x$ \\
\hline 5 & active & active & on & tx, rx \\
\hline
\end{tabular}

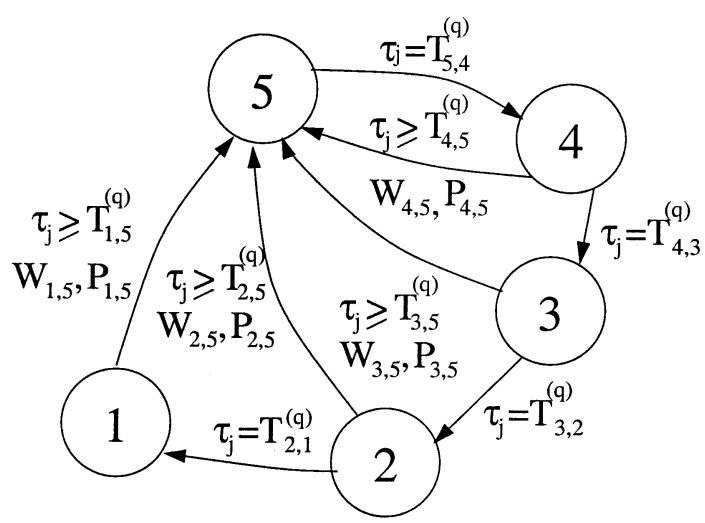

Fig. 3. Example of sleep pattern for a sensor node.

The corresponding sleep pattern evolution is shown in Fig. 3, where arcs connecting the sleep states to state $L=5$ are marked with the associated values of transition delay overhead and power consumption.

\section{B. Paging Scheme}

The objective of the proposed scheme is to page communication nodes that are following their sleep pattern at proper time instants such that the tradeoff between energy saving and delay overhead is optimized.

We assume that the BS knows the service class associated with each node, and for each node in sleep state records the last time instant at which the node was awake (i.e., in state $L$ ). The BS is, therefore, able to predict the current power state of every node under its control. Let us focus on the generic node $j$ belonging to service class $q$. The node's current state, denoted by $l$, is such that the time $\tau_{j}$ elapsed since the last time instant $j$ was in state $L$, is greater than or equal to $T_{l, L}^{(q)}$, but less than the timeout value corresponding to the next deeper power state in the sleep pattern evolution.

We also assume that the BS pages nodes in the same service class by using the same set of paging signals, denoted by $x_{l}^{(q)}(l=1, \ldots, L ; q=1, \ldots, Q)$. The reason for this is that a device in sleep state can detect a paging signal through a passive RAS receiver only if the sequence is transmitted at low rate [18]. Thus, in order to keep the signaling overhead small, paging sequences must be short (namely, 5-8 bits long depending on the technology used), and their number may be not large enough to uniquely address all the nodes in the same area. As a consequence, node $j$ wakes up whenever it has spent in state $l$ a time 


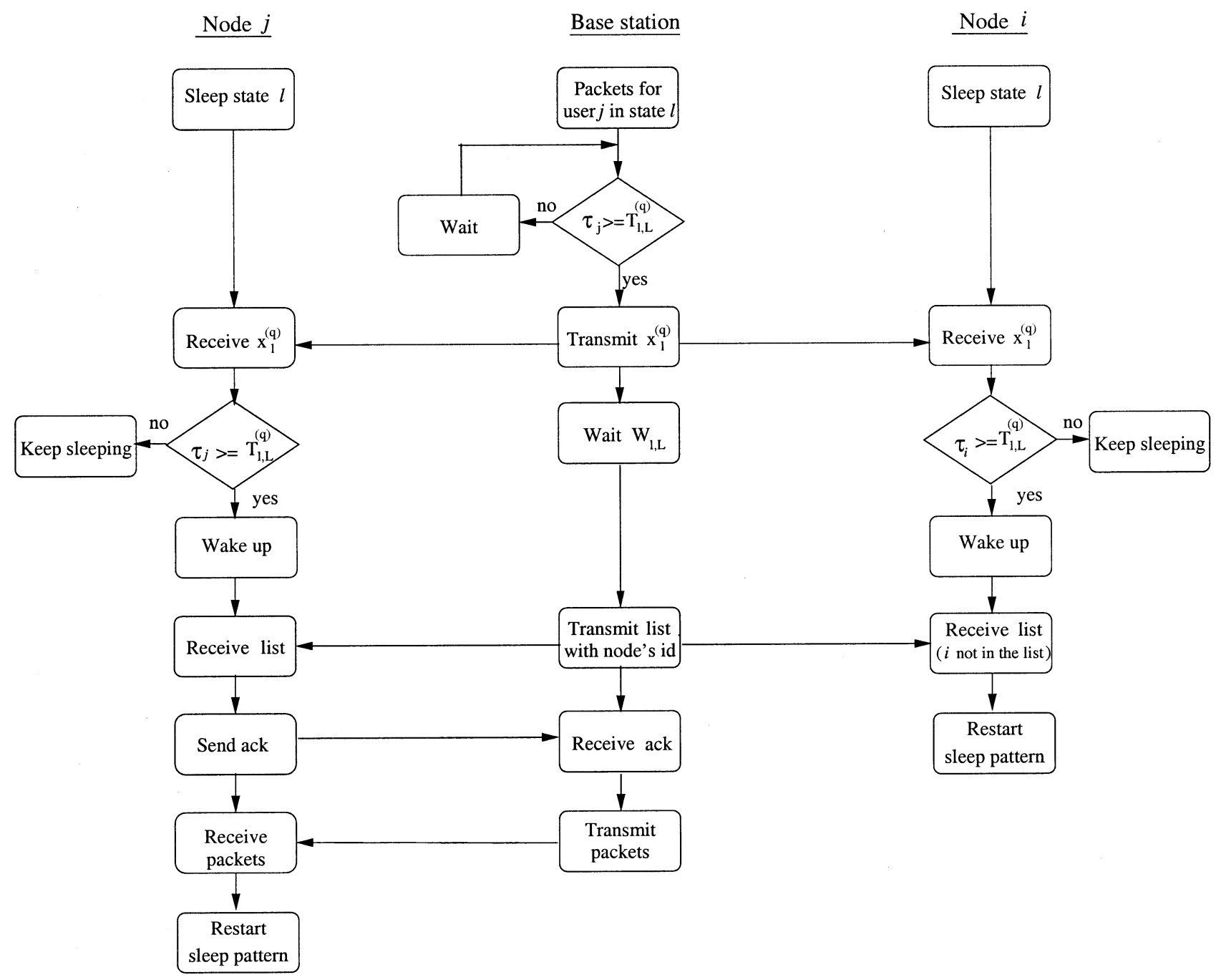

Fig. 4. Diagram of the paging scheme.

equal to or greater than $Y_{l, L}^{(q)}$ (i.e., $\left.\tau_{j} \geq T_{l, L}^{(q)}\right)^{2}$ and either one of these two events takes place: 1) $j$ is intentionally paged by the BS or 2) $j$ detects a paging signal destined to another node which belongs to the same service class and is in the same sleep state.

A diagram of the waking up scheme is shown in Fig. 4, where nodes $i$ and $j$ are assumed to belong to the same service class $q$ and both may be awakened by the same paging signal $x_{l}^{(q)}$. As illustrated in the diagram, node $j$ will receive data packets only if it becomes active upon the paging signal arrival and sends back to the BS an acknowledgment.

Remarks:

1) Note that nodes in the same sleep state do not necessarily all wake up at the same time, nor do they move as a group to the next sleep state although they follow the same sleep pattern.

2) From the diagram in Figs. 4 and 5, one can infer that a paging signal is transmitted by the BS only if the node to be paged will save energy by waking up at that time instant. In fact, the BS pages a node that has pending

${ }^{2}$ Recall that $\tau_{j}$ is the period elapsed since the last time instant $j$ was in state $L$. Thus, by considering (5), the fact that $j$ has spent in state $l$ a time equal to or greater than $Y_{l, L}^{(q)}$ is equivalent to having $\tau_{j} \geq T_{l, L}^{(q)}$.

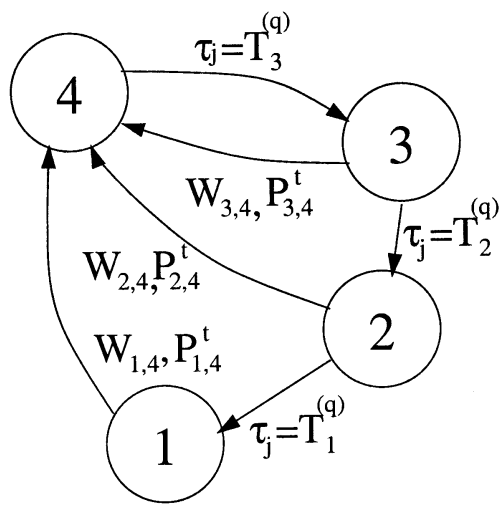

Fig. 5. Evolution of the sleep pattern of reference.

traffic and is currently in sleep state $l$ only if the node has been in $l$ for at least a time period equal to $Y_{l, L}^{(q)}$; otherwise, the paging transmission is delayed.

3) Since the aim of the proposed protocol is to let the nodes be in sleep state as often as possible, the problem of tracking the nodes' location may arise. Consider for example a cellular environment where the geographic area is divided into various location areas, each of them cor- 
responding to a cluster of cells. Let each location area be identified by a local area ID (LAI), as in global system for mobile communication [24]. A node that is not transmitting-receiving packets can determine its current location by periodically listening to the LAI broadcast by the BS and update its position if necessary. In the proposed paging protocol, nodes that are in sleep state and enter a new location area will not update their position, thus, causing an unacceptable increase of signaling traffic and packet delay. To avoid such inefficiencies, the following solution can be implemented. Each BS can transmit an additional signal (namely, a pilot tone at a given frequency or a short bits sequence) to identify the location area to which the BS belongs. Such a signal can be detected by the node through the RAS receiver shown in Fig. 2, so that the node does not need to wake up. All location areas in the network can be locally identified through a small number of different signals by adopting a reuse scheme. Clearly, fast nodes will not significantly benefit from the presented approach since they have to either wake up often, and therefore, they can never reach a deep sleep state, or seldom update their position, hence, suffering the inefficiencies described above. It is our opinion that the fact that fast nodes will consume more energy than slow nodes should be seen as a price to pay for accessing the network services at a higher mobility rate.

\section{Performance Analysis}

Let us focus on one group of $U$ nodes belonging to the same service class and which are assigned the same set of paging sequences and the same sleep pattern. We assume that the time scale is discretized into time slots of unit duration and that the paging signal arrival at the BS is an independent identically distributed (i.i.d.) process with the same distribution for all the nodes in the group. We denote by $P_{a}$ the probability that a paging signal arrives at the BS for a certain node in a time slot; by $\tau_{j}(n)$, the time elapsed from the last time instant the generic node $j$ was awake to time instant $n$, and by $s$ the current power state of the generic node. Also, to make the notation simpler, in the following, we indicate the timeout value after which a sleep state $l(l=1, \ldots, L-1)$ is entered with $T_{l}$, and we replace $Y_{l, L}^{(q)}$ with $Y_{l \cdot{ }^{3}}$

We define $D_{l}$ as the delay from the time instant when a paging signal for the generic node $j$ arrives at the BS to the time instant when the paging signal is transmitted to $j$, conditioned on $j$ being in state $l . D_{l}$ is greater than zero if, at the time instant at which the paging signal for $j$ arrives at the $\mathrm{BS}, \tau_{j}$ is less than $T_{l}+Y_{l}$. We compute the average value of $D_{l}(l=1, \ldots, L-1)$ as

$$
\begin{gathered}
\bar{D}_{l}=\sum_{i=0}^{Y_{l}-1} i \cdot P\{\text { paging for } j \text { arrives at BS when } \\
\left.\tau_{j}=T_{l}+Y_{l}-i \mid s=l\right\}
\end{gathered}
$$

${ }^{3}$ The simplified notation will not cause ambiguity since the precedessor of any sleep state $l(l \neq L)$ is unique, and only one service class is considered here.

$$
\begin{aligned}
& =\sum_{i=0}^{Y_{l}-1} i \cdot P \text { p paging for } j \text { arrives at BS when } \\
& \left.\quad \tau_{j}=T_{l}+Y_{l}-i \mid T_{l} \leq \tau_{j}<T_{f}\right\} \\
& =\sum_{i=0}^{Y_{l}-1} i P_{a}\left(1-P_{a}\right)^{Y_{l}-1-i} .
\end{aligned}
$$

From (7), we find that

$$
\bar{D}_{l}=Y_{l}+P_{a}^{-1}\left[\left(1-P_{a}\right)^{Y_{l}}-1\right] .
$$

By deconditioning on the sleep state assumed by the node and considering that the propagation time over the radio channel is negligible, the total average delay from the time instant when the paging signal has to be transmitted to the time instant when $j$ is awake is

$$
\bar{D}_{t}=\sum_{l=1}^{L-1}\left(\bar{D}_{l}+W_{l, L}\right) \cdot \frac{P\{s=l\}}{\sum_{k=1}^{L} P\{s=k\}} .
$$

In order to compute probability $P\{s=l\}$, we first evaluate the average number of nodes in state $l(l=1, \ldots, L)$. By defining $a^{l}(n)$ as the number of nodes in state $l$ at time instant $n$, we have

$$
\begin{gathered}
a^{l}(n+1)=a^{l}(n)+(\text { no. nodes in } s=b \\
\text { moving to } s=l \text { at time } n) \\
-(\text { no. nodes in } s=l \\
\text { moving to } s=f \text { at time } n) \\
-(\text { no. nodes in } s=l \\
\quad \text { waking up at time } n) .
\end{gathered}
$$

In order to further understand (10), note that: 1) The term (no. nodes in $s=b$ moving to $s=l$ at time $n$ ) is equal to the number of nodes among the $a^{b}(n)$ nodes, whose time spent in sleep state at time $n$ is equal to $T_{l}$, provided that no node is paged in state $b$ at time $n$; otherwise, it is equal to zero. Similarly, for the term (no. nodes in $s=l$ moving to $s=f$ at time $n$ ). 2) The random variables $\tau_{j}(j=1, \ldots, U)$ have the same distribution $\forall j$ since the paging arrival process is i.i.d. and the same for all the nodes. Thus, we can suppress the subscript $j .3$ ) The event that none is paged in state $s$ at time $n$ and that $\tau(n)$ is equal to a given value are independent.

Therefore, we write

$$
\begin{aligned}
& a^{l}(n+1) \\
& =a^{l}(n)+a^{b}(n) P\{\text { none paged in state } s \text { at time } n \mid \\
& s=b\} P\left\{\tau(n)=T_{l} \mid s=b\right\} \\
& -a^{l}(n) P\{\text { none paged in state } s \text { at time } n \mid s=l\} \\
& \quad \cdot P\left\{\tau(n)=T_{f} \mid s=l\right\} \\
& -(\text { no. nodes in } s=l \text { waking up at time } n), \\
& \quad 1 \leq l<L .
\end{aligned}
$$

Equation (11) can be computed by considering that for $1 \leq l<$ $L$, we have

$$
\begin{aligned}
P\{\text { none paged in state } s \text { at time } n \mid s & =l\} \\
& =\left(1-P_{p}^{l}(n)\right)^{a^{l}(n)}
\end{aligned}
$$


where $P_{p}^{l}(n)=P\{$ node paged at time $n \mid s=l\}$ [see the Appendix for the derivation of $\left.P_{p}^{l}(n)\right]$, and

$$
\begin{aligned}
P\left\{\tau(n)=T_{f} \mid s=l\right\} & =P\left\{\tau(n)=T_{f} \mid T_{l} \leq \tau(n)<T_{f}\right\} \\
& =\left(1-P_{a}\right)^{T_{f}-T_{l}-1} .
\end{aligned}
$$

The number of nodes in sleep state $l$ that wake up at time $n$ are the $m$ nodes among the $a^{l}(n)$ nodes that are intentionally paged by the BS $\left(m=1, \ldots, a^{l}(n)\right)$, plus $u$ among the remaining nodes $\left(u=0, \ldots, a^{l}(n)-m\right)$ that have been in state $l$ for a time period equal to or longer than $T_{l}+Y_{l}$. By defining $r_{l}=$ $P\left\{\tau(n) \geq T_{l}+Y_{l} \mid s=l\right\}$ (see the Appendix for the derivation of $r_{l}$ ), we have

$$
\begin{aligned}
& \text { (no. nodes in } s=l \text { waking up at time } n \text { ) } \\
& \left.\begin{array}{rl}
a^{l}(n) a^{l}(n)-m \\
a_{m=1} \sum_{u=0}^{a^{l}(n)}(m+u)\left(P_{p}^{l}(n)\right)^{m} \\
m
\end{array}\right) a^{a^{l}(n)-m} \\
& \quad \cdot\left(1-P_{p}^{l}(n){ }^{m}\right. \\
& \quad \cdot\left(\begin{array}{c}
a^{l}(n)-m \\
u
\end{array}\right)\left(r_{l}\right)^{u}\left(1-r_{l}\right)^{a^{l}(n)-m-u} \\
& =a^{l}(n) P_{p}^{l}+\sum_{m=1}^{a^{l}(n)}\left(\begin{array}{c}
a^{l}(n) \\
m
\end{array}\right)\left(P_{p}^{l}\right)^{m}\left(1-P_{p}^{l}\right)^{a^{l}(n)-m} \\
& \quad \cdot r_{l}\left(a^{l}(n)-m\right) \\
& =a^{l}(n) P_{p}^{l}+a^{l}(n) r_{l}\left[1-\left(1-P_{p}^{l}\right)^{a^{l}(n)}\right]-a^{l}(n) r_{l} P_{p}^{l} .
\end{aligned}
$$

Then, by writing

$$
w^{l}(n)=P_{p}^{l}+r_{l}\left[1-\left(1-P_{p}^{l}\right)^{a^{l}(n)}-P_{p}^{l}\right]
$$

we get

$$
\text { (no. nodes in } s=l \text { waking up at time } n)=a^{l}(n) w^{l}(n)
$$

where $\quad w^{l}(n) \quad$ represents the probability $P\{$ node wakes up at time $n \mid s=l\}$.

Finally, by using (13)-(16) in (11), we obtain

$$
\begin{aligned}
& a^{l}(n+1)=a^{l}(n)+a^{b}(n)\left(1-P_{p}^{b}\right)^{a^{b}(n)}\left(1-P_{a}\right)^{T_{l}-T_{b}-1} \\
& \quad-a^{l}(n)\left(1-P_{p}^{l}\right)^{a^{l}(n)}\left(1-P_{a}\right)^{T_{f}-T_{l}-1}-a^{l}(n) w^{l}(n) .
\end{aligned}
$$

Similarly, for $l=L$, we have

$$
\begin{aligned}
a^{L}(n+1)=a^{L}(n) & -a^{L}(n)\left(1-P_{a}\right) \\
& +\sum_{l=1}^{L-1} a^{l}\left(n-W_{l, L}\right) w^{l}\left(n-W_{l, L}\right) .
\end{aligned}
$$

Notice that the total number of nodes that are in the transition periods $W_{l, f}(l=1, \ldots, L-1$ and $f>l)$ is equal to $U-$ $\sum_{l=1}^{L} a^{l}(n)$.
From (17)-(18), we can derive the average number of nodes in state $l(l=1, \ldots, L)$ as

$$
\overline{a^{l}}=\frac{1}{N} \sum_{n=0}^{N} a^{l}(n)
$$

where $N$ is a sufficiently large integer number. The probability that a node is in state $l$ is

$$
P\{s=l\}=\frac{\overline{a^{l}}}{U} .
$$

By using (9) and (20), we obtain the total average delay from the time instant when the paging signal has to be transmitted to the time instant when the node is awake as

$$
\begin{aligned}
\bar{D}_{t} & =\sum_{l=1}^{L-1}\left\{Y_{l}+P_{a}^{-1}\left[\left(1-P_{a}\right)^{Y_{l}}-1\right]+W_{l, L}\right\} \\
& =\frac{\overline{a^{l}}}{\sum_{k=1}^{L} \overline{a^{k}}} .
\end{aligned}
$$

By knowing the expression of $P_{p}^{l}$ and $P\{s=l\}$, we derive the channel occupation due to the transmission of paging signals as

$$
c_{o}=\sum_{l=1}^{L} P_{p}^{l} \cdot U \cdot e \cdot P\{s=l\}
$$

where $e$ is the paging signal duration.

By considering the generic period of time $t$, we derive the average power saving per node as

$\mathrm{PS}=\frac{1}{t} \sum_{l=1}^{L-1}\{($ power saved in $l) \cdot($ time spent in $l)$

- $[($ power cost of transition from $l$ to $L)$

- (power consumed in $L)] \cdot W_{l, L}$

-(no. times node wakes up while being in $l$ )

- [(power cost of transition from $l$ to $f$ )

- (power consumed in $L)] \cdot W_{l, f}$

.(no. times node moves to $f$ while being in $l$ ) $\}$.

We write

(no. times node wakes up while being in $l$ )

$$
=\sum_{n=0}^{t} \frac{a^{l}(n)}{U} w^{l}(n)
$$

(no. times node moves from $l$ to $f$ )

$$
=\sum_{n=0}^{t} \frac{a^{l}(n)}{U}\left(1-P_{p}^{l}\right)^{a^{l}(n)}\left(1-P_{a}\right)^{T_{f}-T_{l}-1}
$$

therefore

$$
\begin{aligned}
\mathrm{PS}=\frac{1}{t} \sum_{l=1}^{L-1}\{ & \left(P_{L}-P_{l}\right) \cdot t \cdot P\{s=l\}-\left(P_{l, L}-P_{L}\right) \cdot W_{l, L} \\
& \cdot \sum_{n=0}^{t} \frac{a^{l}(n)}{U} w^{l}(n)-\left(P_{l, f}-P_{L}\right) \cdot W_{l, f} \\
& \left.\cdot \sum_{n=0}^{t} \frac{a^{l}(n)}{U}\left(1-P_{p}^{l}\right)^{a^{l}(n)}\left(1-P_{a}\right)^{T_{f}-T_{l}-1}\right\} .
\end{aligned}
$$


TABLE II

Timeout VALUes of the CONSIDERED SLeEP PATTERnS (EXPRESSED IN MiLLISECONDS)

\begin{tabular}{c||c|c|c|c}
\hline & $q=1$ & $q=2$ & $q=3$ & $q=4$ \\
\hline$T_{4,3}^{(q)}$ & 1 & 1 & 1 & 1 \\
\hline$T_{3,2}^{(q)}$ & $10^{3}$ & 50 & 50 & 80 \\
\hline$T_{2,1}^{(q)}$ & $10^{4}$ & 200 & 200 & 300 \\
\hline$Y_{l, 4}^{(q)}$ & $Z_{l, 4}$ & $Z_{l, 4}$ & $Z_{l, 4}+20(4-l)$ & $Z_{l, 4}+60(4-l)$ \\
\hline
\end{tabular}

\section{RESULTS}

Through the analysis carried out in Section V, we derive the performance of the proposed scheme in terms of power gain (denoted by PS) average delay of the paging signals $\bar{D}_{t}$, and channel occupation due to paging.

We assume that the number of nodes is equal to ten and $L$ is equal to four; the considered sleep pattern evolution is shown in Fig. 5. We compute the system parameter values based on the data reported in [12]. We consider that the power consumed at the node in a sleep state is normalized with respect to the power consumed while the node is in receiving mode; thus, we have: $P_{4}=1, P_{3}=0.63, P_{2}=0.31, P_{1}=0.057$, and $P_{l, 4}=(1.2)^{L-l}$ for $l=1, \ldots, 3$. The time to wake up from every sleep state is assumed to be constant and equal to: $W_{3,4}=10 \mathrm{~ms}, W_{2,4}=100 \mathrm{~ms}$, and $W_{1,4}=150 \mathrm{~ms}$. Results were obtained also for uniformly distributed delay overheads with average equal to the above constant values; in this case, $Z_{l, f}(l, f=1, \ldots, 4)$ was derived from (3) by using the average delay overheads. However, no significant difference was observed between the two sets of results.

The results shown in the following are derived by considering four different sleep patterns $\Sigma^{(q)}$ with $q=1, \ldots, 4$, whose parameters are reported in Table II. The parameters $T_{l, 4}^{(q)}(l=$ $1, \ldots, 3)$ can be derived from (5) by using the values in Table II. We note that sleep pattern $\Sigma^{(1)}$ lets nodes remain awake with high probability, while patterns $\Sigma^{(q)}$ with $q=2, \ldots, 4$ allow nodes to enter sleep states more quickly, relatively to $\Sigma^{(1)}$. As the sleep patterns vary from $\Sigma^{(2)}$ to $\Sigma^{(4)}, Y_{l, 4}^{(q)}(l=1, \ldots, 3)$ increases and the time spent by the nodes in deep sleep states is longer. Also, the transition times $T_{l, l-1}^{(q)}(l=2, \ldots, 4)$ are longer for $q=4$ than for $q=2,3$. The values shown in Table II were arbitrary chosen as examples of different operational conditions. The derivation of optimal sleep patterns depending on the required QoS and hardware constraints will be subject of further research.

Fig. 6 presents the behavior of PS and $\bar{D}_{t}$ as the arrival rate of paging signals varies, for sleep pattern $\Sigma^{(2)}$ and $\Sigma^{(3)}$. The plot shows that power saving decreases as paging arrival rate increases because nodes characterized by a higher paging rate enter deep sleep states with lower probability. However, Fig. 6 shows that significant power gains can be achieved even for a high downlink traffic load (i.e., high values of $P_{a}$ ) if a delay in the node's reaction is allowed. On the other hand, if the node has tight delay constraints, a significant power gain can be obtained only in the case of low downlink traffic load. The plot can be used to evaluate the tradeoff between the average delay and the power saving experienced by the nodes as downlink traffic

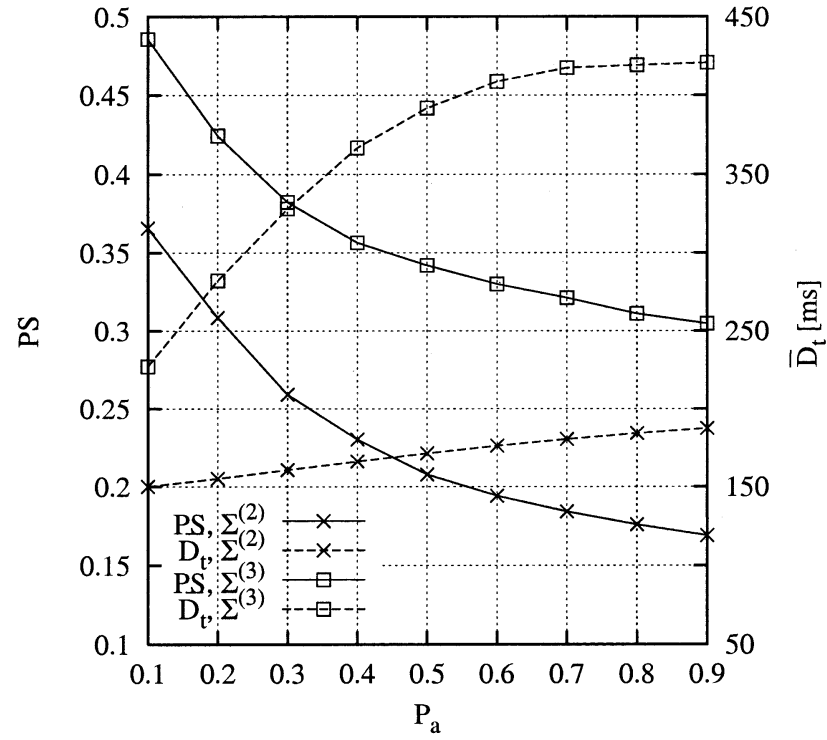

Fig. 6. Power gain and paging delay versus paging signal arrival rate for two different sleep patterns.

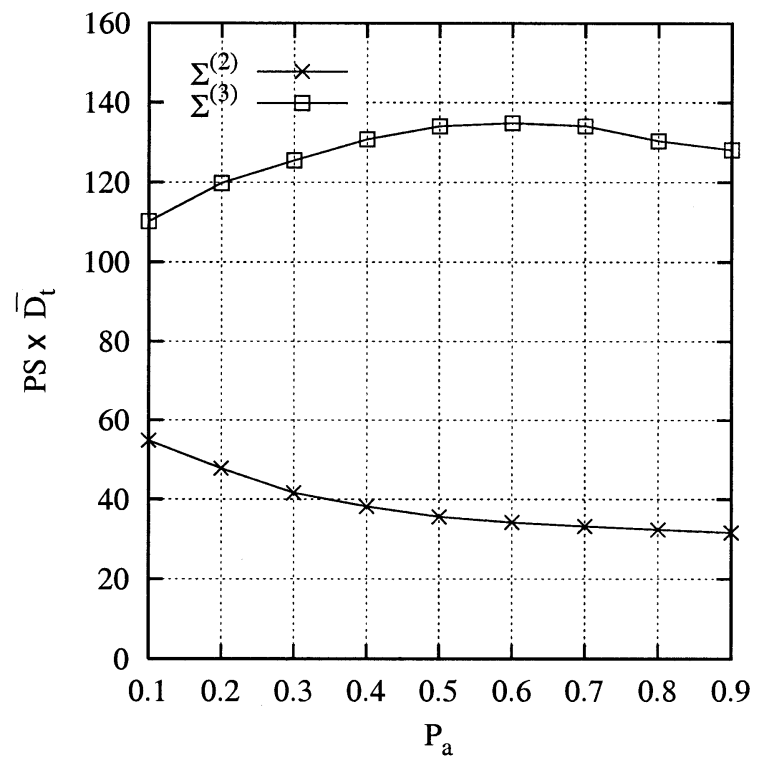

Fig. 7. Power gain-paging delay product versus paging signal arrival rate for two different sleep patterns.

load vary. Similar results are shown in Fig. 7, where the product between the power gain and the paging delay is plotted as a function of the arrival rate of paging signals.

Channel utilization due to paging signals transmitted by the BS is presented in Fig. 8. The paging signal duration $e$ is assumed to be ten times less than the packet transmission time. As we vary the sleep pattern from $\Sigma^{(1)}$ to $\Sigma^{(4)}$, we get higher values for $Y_{l, 4}^{(q)}(l=1, \ldots, 3)$, and nodes are forced to stay longer in the sleep states. In this case, the downlink traffic transmitted by the BS becomes more bursty and, therefore, channel occupation due to paging decreases. For a fixed sleep pattern, the number of paging signals sent by the BS and of acknowledgments sent by the paged nodes increase as the paging signal arrival rate $P_{a}$ increases. 


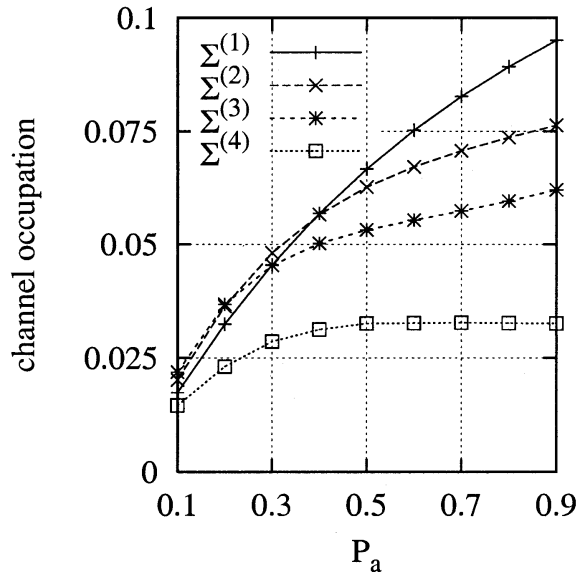

Fig. 8. Channel occupation due to paging signals.

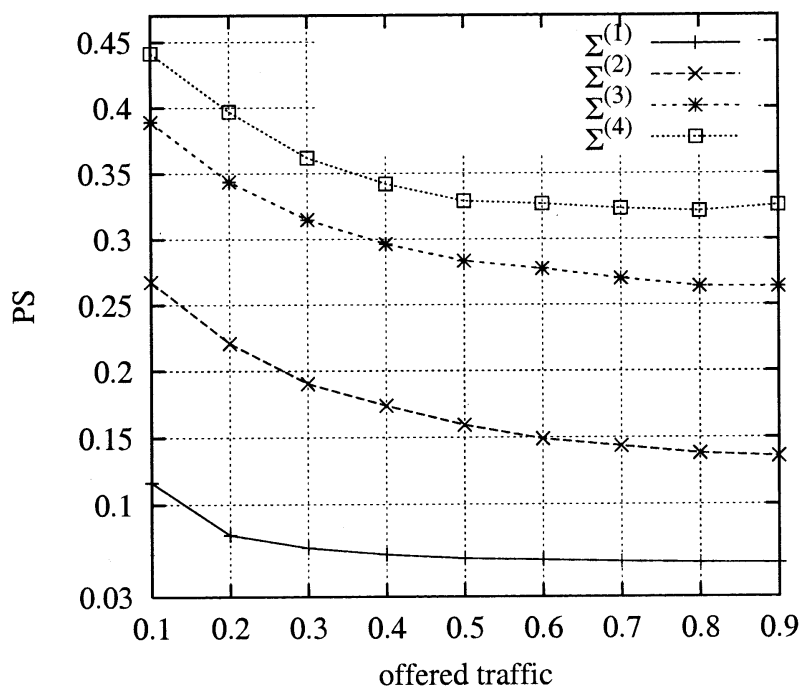

Fig. 9. Power gain obtained through the proposed scheme.

Figs. 9-11 present results obtained through simulation by assuming the same scenario as presented in [9] for comparison purposes. In [9], the downlink packet traffic is assumed to arrive at the BS according to a Poisson distribution. The length of each packet is geometrically distributed and the time unit duration is chosen equal to the mean packet transmission time (we set the mean packet transmission time equal to $10 \mathrm{~ms}$ ). The BS queues the downlink traffic in an infinite buffer and exhaustively serves the node that sent an acknowledgment. If more than one node sent an acknowledgment at the same time, they are served in a random order. (Please, refer to [9] for further details on the simulation scenario.)

Figs. 9 and 10 show the power gain and the mean packet delay, respectively, as the downlink traffic load varies. Packet delay is measured from the time instant when a packet arrives at the BS to the time instant when the packet is delivered to the node. The IB protocol presented in [9] gives a lower packet delay than the proposed solution; in [9], however, the possibility to increase energy savings by entering different sleep states is not considered. (Note, in fact, that in Fig. 9 the performance in terms of power saving is not reported for the IB protocol.) For the proposed approach, we consider different sleep patterns corresponding to in-

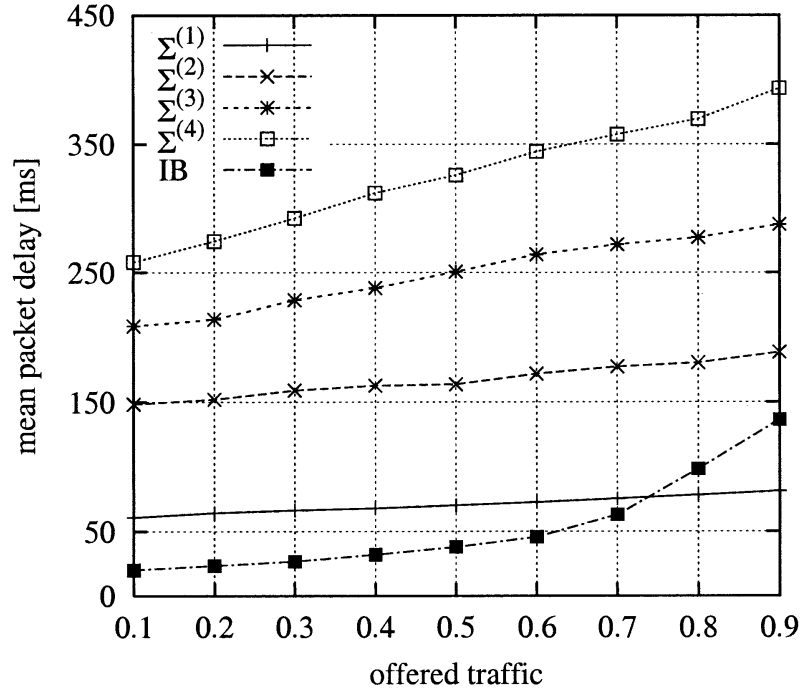

Fig. 10. Mean packet delay due to the proposed scheme compared to the mean packet delay obtained through the IB protocol.

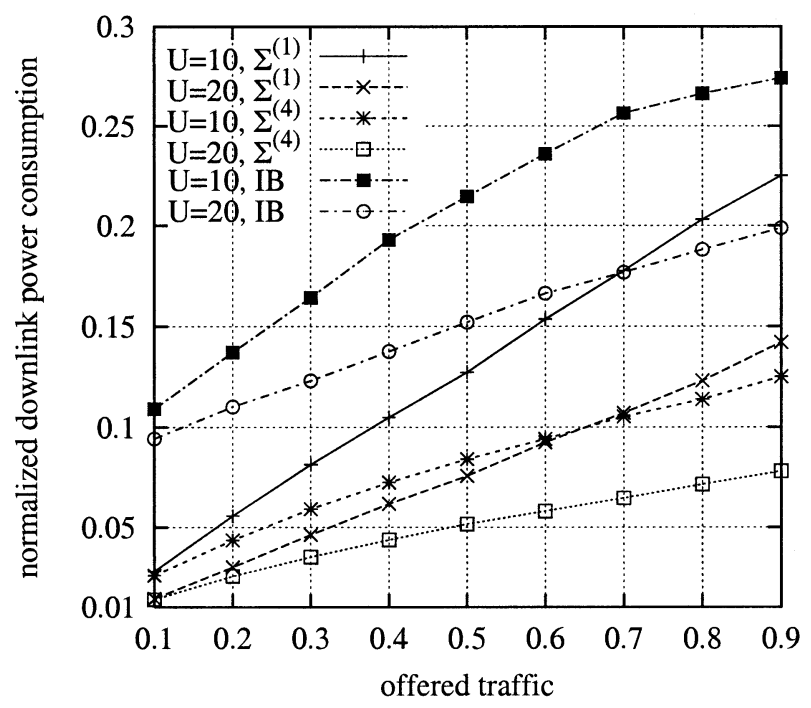

Fig. 11. Normalized downlink power consumption due to the proposed protocol compared to the power consumption due to the IB paging scheme.

creasing timeouts and/or values of $Y_{l, 4}^{(q)}$; thus, we obtain greater power gain and higher packet delay as we pass from $\Sigma^{(1)}$ to $\Sigma^{(4)}$. For a low value of PS (i.e., when pattern $\Sigma^{(1)}$ is used), we achieve a mean packet delay comparable to the results obtained for the IB paging scheme; patterns $\Sigma^{(2)}, \Sigma^{(3)}$, and $\Sigma^{(4)}$ imply a large delay, but also a high power gain.

Fig. 11 shows the average downlink power consumed by a node while being awake, i.e., the average power spent to receive downlink traffic and transmit acknowledgments back to the BS. As in [9], the transmission power is set equal to 100, the paging signal duration $e$ is assumed to be ten times less than the mean packet transmission time, and the acknowledgment duration is set equal to $0.02 \cdot e$. Curves are obtained for a number of nodes equal to 10 and 20 . As the traffic load increases, the number of received packets increases, as well as the number of transmitted acknowledgments; thus, the power consumption grows. When the number of nodes varies from 10 to 20 while the offered traffic is held constant, performance improves since the 


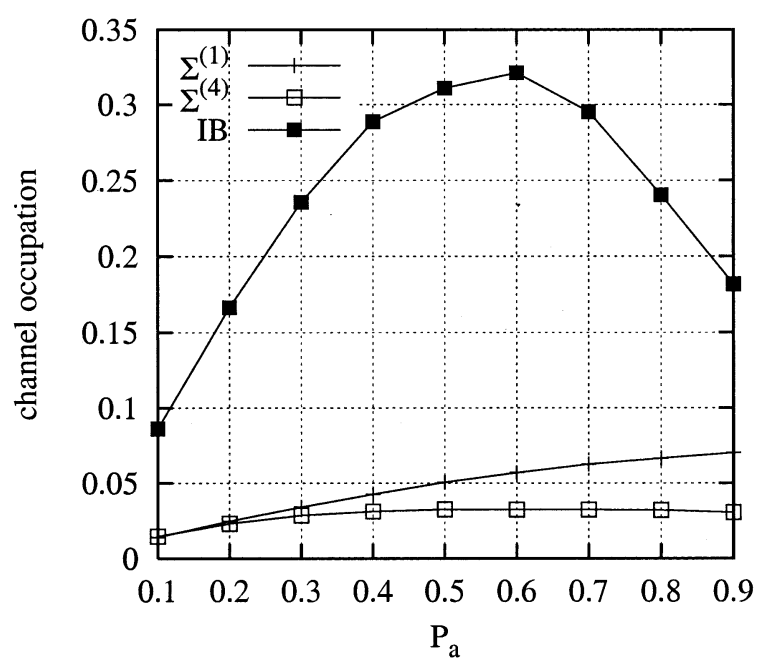

Fig. 12. Channel occupation due to paging signals. The proposed scheme and the IB protocol are compared.

downlink traffic load per node decreases. A comparison with the IB scheme shows that the proposed protocol gives better performance for any of the considered sleep patterns. For $U=10$ and an offered traffic equal to 0.9 , the obtained improvement is roughly equal to $16 \%$ in the case of $\Sigma^{(1)}$, and to $53 \%$ in the case of $\Sigma^{(4)}$. An even greater improvement is achieved for $U=20$. However, as shown in Fig. 10, such a great reduction in power consumption with respect to the IB scheme is achieved at the cost of a much higher mean packet delay.

Finally, Fig. 12 compares the channel occupancy due to the proposed paging protocol with the performance of the IB scheme. The improvement reached with the proposed scheme with respect to the IB scheme is significant for any value of $P_{a}$ and is almost equal to one order of magnitude for $P_{a}$ taking values in the range between 0.3 and 0.8 . The behavior of the channel occupancy due to paging signals in the IB protocol can be explained as follows [9]. As the offered traffic grows, the channel occupancy increases since more paging messages are delivered. However, at a critical value of offered traffic, where the BS pages several users with the same paging message, the channel occupancy starts decreasing. Moreover, paging tends to vanish as the traffic load increases.

\section{CONCLUSION AND DISCUSSION}

In this paper, we have proposed a new paging scheme that allows nodes to wake up only when necessary. The paging protocol is combined with a power management policy maximizing the node power saving while still meeting the QoS requirements on packet delay. Results showing the tradeoff between power gain and traffic delay were obtained through both analysis and simulation. Compared to the IB protocol, the presented scheme performs better in terms of both power consumption and paging channel occupation.

We would like to emphasize that there are several related issues that still need to be solved. For example, with wireless devices supporting a variety of traffic, further work has to be done in order to develop a system that dynamically adapts to the applications. In particular, optimal timeout values for the sleep pat- terns associated with different service classes have to be defined. Also, a probability of error in the BS estimation of the nodes' operational state should be introduced. This would enable a performance study of the proposed scheme in the presence of sleep patterns with probabilistic transitions. Finally, the problem of optimally trading-off between power saving and QoS provisioning for uplink traffic has to be addressed. In this case, each network node should compute its scheduling policy for packet transmission based on the node's QoS requirements, energy constraints, and the network traffic load. The possibility to use stochastic dynamic programming for optimally solving the problem should be explored.

\section{APPENDIX A}

Recall that the arrival of paging signals at the BS is assumed to be an i.i.d. process with the same distribution for all the nodes in the group. For $l=2, \ldots, L-1$, we have

$$
\begin{aligned}
r_{l}= & P\left\{\tau(n) \geq T_{l}+Y_{l} \mid s=l\right\} \\
& \times P\left\{\tau(n) \geq T_{l}+Y_{l} \mid=T_{l} \leq \tau(n)<T_{f}\right\} \\
& =\sum_{i=Y_{l}-1}^{T_{f}-T_{l}-1} P_{a}\left(1-P_{a}\right)^{i}\left(1-P_{a}\right)^{Y_{l}-1}-\left(1-P_{a}\right)^{T_{f}-T_{l}}
\end{aligned}
$$

$$
\begin{aligned}
P_{p}^{l}(n)= & P\{\text { node paged at time } n \mid s=l\} \\
= & P\left\{\text { node paged at time } n \mid \tau(n)<T_{l}+Y_{l}, s=l\right\} \\
& \cdot P\left\{\tau(n)<T_{l}+Y_{l} \mid s=l\right\} \\
& +P\left\{\text { node paged at time } n \mid \tau(n)=T_{l}+Y_{l}, s=l\right\} \\
& \cdot P\left\{\tau(n)=T_{l}+Y_{l} \mid s=l\right\} \\
& +P\left\{\text { node paged at time } \mathrm{n} \mid \tau(n)>T_{l}+Y_{l}, s=l\right\} \\
& \cdot P\left\{\tau(n)>T_{l}+Y_{l} \mid s=l\right\} \\
= & 0+\sum_{i=1}^{Y_{l}}\left(\begin{array}{c}
Y_{l} \\
i
\end{array}\right) P_{a}^{i}\left(1-P_{a}\right)^{Y_{l}-i} \\
& \cdot P\left\{\tau(n)=T_{l}+Y_{l} \mid T_{l} \leq \tau(n)<T_{f}\right\} \\
& +P_{a} \cdot P\left\{\tau(n)>T_{l}+Y_{l} \mid T_{l} \leq \tau(n)<T_{f}\right\} \\
= & {\left[1-\left(1-P_{a}\right)^{Y_{l}}\right] \frac{1}{T_{f}-T_{l}}+P_{a} \frac{T_{f}-\left(T_{l}+Y_{l}+1\right)}{T_{f}-T_{l}} . }
\end{aligned}
$$

\section{REFERENCES}

[1] I. Chlamtac, C. Petrioli, and J. Redi, "Energy-conserving access protocols for identification networks," IEEE/ACM Trans. Networking, vol. 7, pp. 51-59, Feb. 1999.

[2] M. Flament et al., "A MAC algorithm for energy-limited ad-hoc networks," in Proc. IEEE VTS Fall Vehicular Technology Conf., Sept. 2000, pp. 219-222.

[3] J.-C. Chen, K. Sivalingam, and P. Agrawal, "Performance comparison of battery power consumption in wireless multiple access protocols," ACM/Baltzer Wireless Networks J., vol. 5, no. 6, pp. 445-460, 1999.

[4] Y.-B. Lin, "Paging systems: Network architecture and interfaces," IEEE Network, vol. 11, no. 4, pp. 56-61, July/Aug. 1997.

[5] A. S. Hon. (1993) An Introduction to Paging-What It Is and How It Works. Motorola Electronics Pte Ltd.. [Online]. Available: http://www.motorola.com/MIMS/MSPG/Special/explain_paging/ptoc.html

[6] A. K. Salkintzis and C. Chamzas, "Mobile packet data technology: An insight into MOBITEX architecture," IEEE Pers. Commun., vol. 4, pp. 10-18, Feb. 1997. 
[7] H. Woesner, J.-P. Ebert, M. Schlager, and A. Wolisz, "Power-saving mechanisms in emerging standards for wireless LANs: The MAC level perspective," IEEE Pers. Commun., vol. 5, pp. 40-46, June 1998.

[8] High Performance Radio Local Area Network (HIPERLAN) Type 1, Functional Specification, ETS 300 652, 1996.

[9] A. K. Salkintzis and C. Chamzas, "An in-band power-saving protocol for mobile data networks," IEEE Trans. Commun., vol. 46, pp. 1194-1205, Sept. 1998.

[10] M. Flament et al., "An approach to 4th generation wireless infrastructures-Scenarios and key research issues," in Proc. VTC'99, Houston, TX, May 1999, pp. 16-20.

[11] C.-H. Hwang and A. C.-H. Wu, "A predictive system shutdown method for energy saving of event-driven computation," in IEEE/ACM Int. Conf. Computer-Aided Design, San Jose, CA, Nov. 1997, pp. 28-32.

[12] T. Simunic, L. Benini, and G. De Micheli, "Event-driven power management of portable systems," in Proc. 12th Int. Symp System Synthesis, San Jose, CA, Nov. 1999, pp. 18-23.

[13] Y.-H. Lu, E.-Y. Chung, T. Simunic, L. Benini, and G. De Micheli, "Quantitative comparison of power management algorithms," in Proc. Design, Automation, and Test Eur. Conf., 2000, pp. 49-54.

[14] T. Simunic, H. Vikalo, P. Glynn, and G. De Micheli, "Energy efficient design of portable wireless systems," in Proc. 2000 Int. Symp. Low Power Electronics and Design, 2000, pp. 49-54.

[15] T. Simunic, L. Benini, and G. De Micheli, "Dynamic power management of portable systems," in Proc. MobiCom 2000, Boston, MA, Aug. 2000, pp. 49-54.

[16] A. Sinha and A. P. Chandrakasan, "Dynamic power management in wireless sensor networks," IEEE Des. Test Comput., vol. 18, pp. 62-74, Mar./Apr. 2001

[17] U. Kaiser and W. Steinhagen, "A low-power transponder IC for highperformance identification systems," IEEE J. Solid-State Circuits, vol. 30, pp. 306-310, Mar. 1995

[18] D. Friedman, H. Heinrich, and D.-W. Duan, "A low-power CMOS integrated circuit for filed-powered radio frequency identification tags," in Proc. IEEE Int. Solid-State Circuits Conf., San Francisco, CA, Feb. 1997, pp. 294-296.

[19] R. R. Buted, "Zero bias detector diodes for the RF/ID market," HewlettPackard J., vol. 46, no. 6, pp. 94-98, Dec. 1995.

[20] H. K. Heinrich et al., "Method of Transporting Radio Frequency Power to Energize Radio Frequency Identification Transponders," U.S. Patent 5850 181, Dec. 1998.

[21] H. K. Heinrich et al., "Radio Frequency Identification Transponder with Electronic Circuit Enabling/Disabling Capability,” U.S. Patent 5874 902, Feb. 1999.

[22] M. H. Singer, D. Telli, and A. Kobrinetz, "Personal Locator System," U.S. Patent 5485 163, Jan. 1996.
[23] L. Benini, A. Bogliolo, G. A. Paleologo, and G. De Micheli, "Policy optimization for dynamic power management," IEEE Trans. ComputerAided Design, vol. 18, pp. 813-833, June 1999.

[24] J. Eberspächer and H.-J. Vögel, GSM: Switching, Services and Protocols. New York: Wiley, 1999.

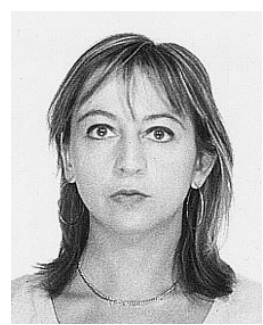

Carla-Fabiana Chiasserini (S'98-M'00) received the electrical engineering degree (summa cum laude) from the University of Florence, Florence, Italy, in 1996 and the Ph.D. degree from the Politecnico di Torino, Torino, Italy, in 1999.

Since 1999, she has been with the Department of Electrical Engineering at Politecnico di Torino, where she is currently an Assistant Professor. She was at the Center for Wireless Communications, University of California, San Diego, CA, as a Visiting Researcher from 1999 to 2001. Her research interests include architectures, protocols, and performance analysis of wireless networks for integrated multimedia services.

Ramesh R. Rao (M'85-SM'90) received the Bachelor's degree in electrical and electronics engineering from the University of Madras, Madras, India, in 1980, and the M.S. and Ph.D. degrees from the University of Maryland, College Park, in 1982 and 1984, respectively.

Since 1984, he has been on the faculty of the Department of Electrical and Computer Engineering at the University of California, San Diego (UCSD), where he is currently Professor and Director of the San Diego Division of the California Institute of Telecommunications and Information Technology. Prior to this appointment, he served as the Director of the UCSD Center for Wireless Communications and was the Vice Chair of Instructional Affairs in the Department of Electrical and Computer Engineering. His research interests include architectures, protocols, and performance analysis of wireless, wireline, and photonic networks for integrated multimedia services. He served as the Editor of the INFORMATION THEORY SOCIETY NEWSLETTER from 1993 to 1995 and is the founding Web Editor of the Information Theory Society web site.

Dr. Rao was elected to the IEEE Information Theory Society Board of Governors (from 1997 to 1999 and 2000 to 2002). He is the Editor for Packet Multiple Access of the IEEE TRANSACTIONS ON COMMUNICATIONS and is a member of the Editorial Board of the ACM/BALTZER WIRELESS NETWORK JOURNAL, as well as IEEE Network Magazine. 